\title{
Universal Statistics of Transport in Disordered Conductors
}

\author{
Hyunwoo Lee ${ }^{1}$, A. Yu. Yakovetz ${ }^{2}$, and L. S. Levitov ${ }^{1,2}$ \\ ${ }^{1}$ Department of Physics, Massachusetts Institute of Technology, \\ 77 Massachusetts Ave., Cambridge, MA 02139, \\ ${ }^{2}$ Landau Institute for Theoretical Physics, \\ 2 Kosygin St., Moscow 117334, Russia
}

\begin{abstract}
In low temperature limit, we study electron counting statistics of a disordered conductor. We derive an expression for the distribution of charge transmitted over a finite time interval by using a result from the random matrix theory of quasi one dimensional disordered conductors. In the metallic regime, we find that the peak of the distribution is Gaussian and shows negligible sample to sample variations. We also find that the tails of the distribution are neither Gaussian nor Poisson and exhibit strong sample to sample variations.
\end{abstract}

PACS numbers: 72.10.Bg, 73.50.Fq, 73.50.Td

Typeset using REVTEX 
The physics of current fluctuations at low temperature presents an interesting quantum mechanical problem. Classical Johnson-Nyquist noise formula [1] gives a good description of current fluctuations due to thermal fluctuations. However, at low temperature thermal fluctuations are small and a new type of noise becomes important. At low temperature, the quantum nature of the current and the discreteness of electron charge is the main source of current fluctuations and due to these reasons, this noise is called "quantum shot noise".

Lots of the low temperature current fluctuation studies deal with a disordered conductor because it has a simple and well established mathematical description based on Landauer's approach [2]. Lesovik [3] and Yurke and Kochanski [4] studied the quantum shot noise in a two-terminal conductor using this approach and found an expression for noise power which is a factor $1-T$ off the classical shot noise, where $T$ is a transmission coefficient. This analysis was generalized to a multiterminal conductor by Büttiker [5], and he also found the reduction of the noise. Physically, the noise reduction is due to the Fermi statistics that leads to correlation of transmission events.

In Landauer's approach, details of current transport are determined by transmission coefficients and there have been many works on the distribution of the coefficients. In the past decade, the random matrix theory of disordered conductors was developed [6], motivated by theoretical discovery and experimental observation of the universal conductance fluctuations. The theory succeeded in providing a complete characterization of the distribution and it also succeeded in providing the insight on the origin of the universal conductance fluctuations. One of the fundamental results in the random matrix theory is the universality of the distribution in the metallic regime and the universality provides a link between the microscopically calculated transmission coefficients and the macroscopically measurable conductance.

Application of the universal distribution to current fluctuations also provides insights on the current fluctuations of a disordered conductor. Beenakker and Büttiker [7] calculated the sample averaged noise power using the universal distribution and found that it depends only on the conductance and that it is one-third of the classical value. 
Noise power is a good measure of noise magnitude and its study revealed the reduction of noise due to Fermi correlation. However, compared to Johnson-Nyquist noise, our understanding of quantum shot noise is limited and not many things are known besides the noise magnitude. Our goal in this paper is to explore the physics of low temperature current fluctuations beyond the scope of noise power. For our purpose, it is useful to look at the behavior of current fluctuations in the time domain, which brings one to the notion of counting statistics of charge transmitted in a conductor over fixed time. A previous study of the counting statistics for a single channel conductor revealed that the attempts to transmit electrons are highly correlated and almost periodic in time, which leads to binomial statistics [8].

In the time domain study of current fluctuations, the charge $Q(t)$ (measured in the units of $e$ ) transmitted over a time interval $t$ is the quantity of interest and the probability distribution $P(Q(t))$ reflects the nature of the current fluctuations. Even at zero temperature, $P(Q(t))$ has finite peak width due to the quantum nature of current. To study $P(Q(t))$, it is useful to introduce the characteristic function $\chi(\lambda)$,

$$
\chi(\lambda)=\sum_{\text {integer } Q} e^{i Q \lambda} P(Q) \text { for }-\pi<\lambda<\pi,
$$

because in many cases, $\chi(\lambda)$ is easier to calculate than $P(Q(t))$ itself. $\chi(\lambda)$ is a Fourier transform of $P(Q(t))$ and so once $\chi(\lambda)$ is obtained, we can either take an inverse Fourier transform of it to get an explicit expression for $P(Q(t))$, or expand it as follows to get cumulants of the distribution:

$$
\log \chi(\lambda)=\sum_{k=1}^{\infty} \frac{(i \lambda)^{k}}{k !}\left\langle\left\langle Q^{k}\right\rangle\right\rangle .
$$

In the linear transport regime, we derive a general expression for $\chi(\lambda)$ in terms of transmission coefficients and by combining it with the transmission coefficients distribution for quasi one dimensional conductors, we show

$$
\overline{\log \chi(\lambda)}=\frac{G V t}{e} \operatorname{arcsinh}^{2} \sqrt{e^{i \lambda}-1},
$$


where $V$ is the dc voltage, $G=g\left(e^{2} / h\right)=(N l / L)\left(e^{2} / h\right)$ is the average conductance, and the bar on the left hand side represents the sample average. Cumulant expansion of Eq. (3) implies that in average, for $G V t / e \gg 1, P(Q(t))$ has a Gaussian peak at GVt/e with $\left\langle\left\langle Q^{2}(t)\right\rangle\right\rangle=G V t / 3 e$. It also implies that even though the peak is Gaussian, the tails show deviation from both Gaussian and Poisson distributions. We estimate sample to sample variations of $P(Q(t))$ by studying variances of various quantities and find that for $G V t / e \gg 1$ sample to sample variations of $P(Q(t))$ appear only in the tails of $P(Q(t))$ and that around the peak, $P(Q(t))$ is universal.

Before we present the derivation of above result, we stress that there are two kinds of averages involved. To avoid confusion, we will use a bar $(\cdots)$ for an ensemble average, or an average over samples, and a bracket $(\langle\cdots\rangle)$ for a quantum average, or a quantum expectation value. Also we reserve a double bracket $(\langle\langle\cdots\rangle\rangle)$ for a cumulant of a quantum expectation value and "var" $(\operatorname{var}(\cdots))$ for $\overline{\cdots^{2}}-\bar{\cdots}^{2}$.

Now, let us derive Eq. (3). Following the Landauer's approach [2], we consider a conductor sandwiched between two perfect leads. In the linear transport regime, scattering properties of a conductor are described by a unitary scattering matrix $\hat{S}$ that relates incoming and outgoing amplitudes, $I_{L(R)}$ and $O_{L(R)}$ :

$$
\hat{S}\left(\begin{array}{c}
I_{L} \\
I_{R}
\end{array}\right)=\left(\begin{array}{c}
O_{L} \\
O_{R}
\end{array}\right),
$$

where the subscripts $L$ and $R$ stand for the left and the right leads.

The unitarity of $\hat{S}$ is due to the current conservation, and it allows a system to be decomposed into independent channels [9]. Then the decomposition motivates one to study single channel transport first, where a transmission coefficient $T$ determines the transport. Recently, the counting statistics of the single channel transport was studied [8]. In the low temperature limit $\left(k_{B} \mathcal{T} \ll e V\right)$, the characteristic function $\chi_{1}(\lambda)$ of a single channel system becomes

$$
\chi_{1}(\lambda)=\left(p e^{i \lambda}+q\right)^{M}
$$


where $p=T, q=1-T, M=e V t / h$ [10] and $M \gg 1$ is assumed. The inverse Fourier transform of Eq. (5) gives the binomial distribution, which implies that the intervals between subsequent attempts to transmit electrons are quite regular. This regularity is due to Pauli exclusion principle.

Having the characteristic function of a single channel, we write the total characteristic function $\chi(\lambda)$ as a product,

$$
\chi(\lambda)=\prod_{j}\left(T_{j} e^{i \lambda}+1-T_{j}\right)^{M},
$$

where $T_{j}$ is a transmission coefficient of channel $j$. The product form Eq. (6) follows from the mutual independence of channels. By taking logarithm of Eq. (6), we get

$$
\log \chi(\lambda)=M \sum_{j} \log \left(T_{j} e^{i \lambda}+1-T_{j}\right),
$$

and by expanding Eq. (7) in terms of $\lambda$, we find

$$
\left\langle\left\langle Q^{k}(t)\right\rangle\right\rangle=\left.M \sum_{j}\left(T(1-T) \frac{d}{d T}\right)^{k-1} T\right|_{T=T_{j}} .
$$

We note that both $\log \chi(\lambda)$ and $\left\langle\left\langle Q^{k}(t)\right\rangle\right\rangle$ are linear statistics of $T_{j}$ 's.

Current fluctuations are determined by distribution of transmission coefficients and the distribution varies from sample to sample even though samples have the same macroscopic parameters. Therefore in principle each sample exhibits distinctive current fluctuations. However according to the random matrix theory of disordered conductors, in the metallic regime $(1 \ll g \ll N)$ where $N$ is the number of channels, the distribution approaches a universal one [6]. This result provides a motivation to approximate the sample-dependent distribution by the universal one. To exploit the universal distribution, we introduce new variables $\nu_{j}$ 's and the density function $D(\nu)$ defined by $T_{j}=1 / \cosh ^{2} \nu_{j}$ and $D(\nu) d \nu=$ $D(T) d T$, where $D(T)$ is the density function of $T_{j}$ 's. According to Ref. [6], $D(\nu)$ is uniform over a wide range of $\nu$,

$$
D(\nu)=g \text { for } \nu<\nu_{c}
$$


We combine Eq. (7) with the universal distribution to obtain

$$
\overline{\log \chi(\lambda)}=Q_{0} \int_{0}^{\infty} d \nu \log \left(\frac{e^{i \lambda}-1}{\cosh ^{2} \nu}+1\right),
$$

where $Q_{0}=g M$. In Eq. (10) the upper limit $\nu_{c}$ is replaced by infinity, which is valid in the metallic regime because for large $\nu$, the integrand is exponentially small. The evaluation of the integral then leads to Eq. (3). We note that because $\log \chi(\lambda)$ is a linear statistic, the universal distribution approximation is equivalent to taking an average over samples.

Cumulants are useful in understanding features of the probability distribution. By using the formula Eq. (2), we obtain sample averaged cumulants

$$
\begin{array}{rlrl}
\overline{\langle\langle Q(t)\rangle\rangle}=Q_{0}, & \overline{\left\langle\left\langle Q^{2}(t)\right\rangle\right\rangle}=\frac{1}{3} Q_{0}, \\
\overline{\left\langle\left\langle Q^{3}(t)\right\rangle\right\rangle}=\frac{1}{15} Q_{0}, & \overline{\left\langle\left\langle Q^{4}(t)\right\rangle\right\rangle}=-\frac{1}{105} Q_{0}, \\
\overline{\left\langle\left\langle Q^{5}(t)\right\rangle\right\rangle}=-\frac{1}{105} Q_{0}, & \overline{\left\langle\left\langle Q^{6}(t)\right\rangle\right\rangle}=\frac{1}{231} Q_{0}, \\
\overline{\left\langle\left\langle Q^{7}(t)\right\rangle\right\rangle}=\frac{27}{5005} Q_{0}, & \overline{\left\langle\left\langle Q^{8}(t)\right\rangle\right\rangle}=-\frac{3}{715} Q_{0}, \\
\overline{\left\langle\left\langle Q^{9}(t)\right\rangle\right\rangle}=-\frac{233}{36465} Q_{0}, \overline{\left\langle\left\langle Q^{10}(t)\right\rangle\right\rangle}=\frac{6823}{969969} Q_{0}, \cdots .
\end{array}
$$

The first cumulant is trivial. It is just a definition of $G$ and it shows where the peak of $P(Q(t))$ is. The second cumulant measures the width square of the peak. It is also directly related to the noise power $P=\int d t\langle\langle I(0) I(t)\rangle\rangle$, a widely used measure of noise magnitude, by $\left\langle\left\langle Q^{2}(t)\right\rangle\right\rangle=t P$ for large $t$, and its ensemble average is one-third of the classical value $Q_{0}$, as first pointed out by Beenakker and Büttiker [7]. The third and the fourth cumulants are measures of skewness and sharpness of the peak, respectively and they are related to 3 and 4-point current current correlation functions by similar relations. We note that all

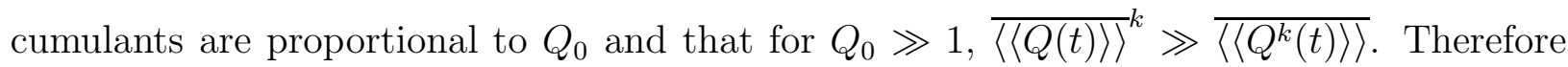
the peak of the distribution $P(Q(t))$ is Gaussian for large conductance limit or long time limit. This result is quite expected from the central limit theorem. Now to see the tails of $P(Q(t))$, we study higher order cumulants. From Eq. (10), we obtain a general formula for the ensemble averaged $k$-th order cumulants

$$
\overline{\left\langle\left\langle Q^{k}(t)\right\rangle\right\rangle}=-i^{k} \frac{Q_{0}}{4} \int_{-\infty}^{\infty} d x \frac{1}{\sqrt{1+e^{-x}}} \int_{-\infty}^{\infty} d q e^{-i q x} \frac{q^{k-1}}{\sinh (\pi q-i 0+)}
$$


and by using the steepest descent method twice, we obtain the asymptotics for large $k$,

$$
\overline{\left\langle\left\langle Q^{k}(t)\right\rangle\right\rangle} \sim \frac{Q_{0}}{(2 \pi)^{k-1}} \frac{(k-1) !}{\sqrt{k}}\left\{\begin{array}{ll}
(-1)^{\frac{k+2}{2}} & \text { for even } k, \\
(-1)^{\frac{k+1}{2}} & \text { for odd } k .
\end{array}\right\}
$$

The high order cumulants diverge as factorial, which suggests that at the tails, $P(Q(t))$ is different from both Gaussian distribution and Poisson distribution which describes the classical current fluctuations. In comparison, $\overline{\left\langle\left\langle Q^{k}(t)\right\rangle\right\rangle}=0$ for $k \geq 2$ for Gaussian distribution and $\overline{\left\langle\left\langle Q^{k}(t)\right\rangle\right\rangle}=Q_{0}$ for $k \geq 1$ for Poisson distribution.

It is known that in the presence of time reversal symmetry, there are order $M$ corrections to $\overline{\langle\langle Q(t)\rangle\rangle}$ and $\overline{\left\langle\left\langle Q^{2}(t)\right\rangle\right\rangle}$ due to weak localization [11], and it is legitimate to expect the same kind of corrections to higher order cumulants. However, because we are interested in the metallic regime, these corrections are small by a factor $g$ and we will ignore them.

A proper next step is to estimate the magnitude of sample to sample variations of $P(Q(t))$. Here instead of $\log \chi(\lambda)$, we examine variance of $\left\langle\left\langle Q^{k}(t)\right\rangle\right\rangle$ to see the variations of $P(Q(t))$. $\left\langle\left\langle Q^{k}(t)\right\rangle\right\rangle$ is a linear statistic and the general formula for the variance of a linear statistic $A=\sum_{j} a\left(T_{j}\right)$ is obtained recently by Beenakker and Rejaei [12], and Chalker and Mecêdo [13],

$$
\begin{array}{r}
\operatorname{var}(A)=\frac{1}{\beta \pi^{2}} \int_{0}^{\infty} d k \frac{k \tilde{a}^{2}(k)}{1+\operatorname{coth}\left(\frac{1}{2} \pi k\right)}, \\
\tilde{a}(k)=2 \int_{0}^{\infty} d \nu a\left(\frac{1}{\cosh ^{2} \nu}\right) \cos k \nu,
\end{array}
$$

where $\beta$ is a symmetry constant, 1,2 , or 4 depending on the symmetry. We use this formula to obtain

$$
\operatorname{var}(\langle\langle Q(t)\rangle\rangle)=\frac{2}{15 \beta} M^{2}, \operatorname{var}\left(\left\langle\left\langle Q^{2}(t)\right\rangle\right\rangle\right)=\frac{46}{2835 \beta} M^{2}, \operatorname{var}\left(\left\langle\left\langle Q^{3}(t)\right\rangle\right\rangle\right)=\frac{11366}{1447875 \beta} M^{2}, \cdots
$$

We note that for low order cumulants, ${\overline{\left\langle\left\langle Q^{k}(t)\right\rangle\right.}}^{2}$ is larger than $\operatorname{var}\left(\left\langle\left\langle Q^{k}(t)\right\rangle\right\rangle\right)$ by at least a factor of $g^{2}$, which is large in the metallic regime. Low order cumulants decide the shape of $P(Q(t))$ around the peak and therefore the small variance of low order cumulants implies that the peak shape shows little sample to sample variations, that is, it is almost universal. 
To see the behavior of higher order cumulants, we obtain an asymptotic form of the variance from an approximate variance formula in Ref. [14],

$$
\operatorname{var}\left(\left\langle\left\langle Q^{k}(t)\right\rangle\right\rangle\right) \sim \frac{4(2 k-1) !}{(2 \pi)^{2 k} \beta} M^{2} .
$$

According to Eq. (117), for high order cumulants, $\operatorname{var}\left(\left\langle\left\langle Q^{k}(t)\right\rangle\right\rangle\right)$ becomes larger than ${\overline{\left\langle\left\langle Q^{k}(t)\right\rangle\right.}}^{2}$ due to its rapidly growing factorial factor, which suggests that the tails of $P(Q(t))$ show large sample to sample variations. We argue that this rapid growth of $\operatorname{var}\left(\left\langle\left\langle Q^{k}(t)\right\rangle\right\rangle\right)$ is not an artifact of the approximate variance formula used above because it assumes stronger spectral rigidity than the formula Eq. (14,15) and it has a tendency to slightly underestimate variances. Therefore large sample to sample variations at the tails of $P(Q(t))$ obtained above is not an artifact of the approximation.

In the above, we derived the shape of $P(Q(t))$ by examining $\overline{\log \chi(\lambda)}$ and its cumulant expansion instead of $\log \overline{\chi(\lambda)}$, which might be an intuitively more appropriate ensemble average because it is directly related to $\overline{P(Q(t))}$. However we argue that in contrast to the intuition, $\overline{\log \chi(\lambda)}$ is an appropriate ensemble average for the study of current fluctuations. One reason is that as we remarked earlier, a $k$-point current current correlation function is linearly related to $\left\langle\left\langle Q^{k}(t)\right\rangle\right\rangle$, whose ensemble average can be obtained from $\overline{\log \chi(\lambda)}$ by a simple expansion. Another reason is that as we show later, $\log \overline{\chi(\lambda)}$ either becomes identical to $\overline{\log \chi(\lambda)}$ at short time limit, or is dominated by the conductance fluctuations instead of the current fluctuations.

Calculation of $\overline{\chi(\lambda)}$ is not simple because $\chi(\lambda)$ is not a linear statistic. Muttalib and Chen [15] did this calculation recently by large $N$ limit continuum approximation and showed that at long time limit, $\log \overline{\chi(\lambda)}$ becomes quite different from $\overline{\log \chi(\lambda)}$. Here we present improved calculation by a perturbation method and we believe that our calculation clarifies the reason why two averages become quite different at long time limit.

Because $\chi(\lambda)$ is not a linear statistic, we need joint probability distribution of transmission coefficients to average it over ensembles. After standard variable change, $T=1 /(1+x)$, the joint probability distribution $P(\{x\})$ is 


$$
P(\{x\})=\exp \left(\beta \sum_{a<b} V\left(x_{a}, x_{b}\right)+\beta \sum_{a} U\left(x_{a}\right)\right) .
$$

We choose $V(x, y)=(1 / 2) \log (x-y)+(1 / 2) \log \left(\operatorname{arcsinh}^{2} \sqrt{x}-\operatorname{arcsinh}^{2} \sqrt{y}\right)$ and $U(x)=$ $g \operatorname{arcsinh}^{2}(\sqrt{x})$ based on the exact calculation of the joint probability distribution function for $\beta=2$ by Beenakker and Rejaei [12]. Then,

$$
\begin{gathered}
\overline{\chi(\lambda)}=\frac{Z_{M}}{Z_{0}} \\
Z_{M}=\int \prod_{a} d x_{a} \exp \left(\beta \sum_{a<b} V\left(x_{a}, x_{b}\right)+\beta \sum_{a} U\left(x_{a}\right)+\sum_{a} M \log \frac{x_{a}+e^{i \lambda}}{x_{a}+1}\right) \\
Z_{0}=\int \prod_{a} d x_{a} \exp \left(\beta \sum_{a<b} V\left(x_{a}, x_{b}\right)+\beta \sum_{a} U\left(x_{a}\right)\right) .
\end{gathered}
$$

By expanding $\log \overline{\chi(\lambda)}$ in terms of $M$, we find

$$
\log \overline{\chi(\lambda)}=\overline{\log \chi(\lambda)}+\operatorname{var}(\log \chi(\lambda))+O\left(M^{3}\right)
$$

and from the formula Eq. (14, 15), we obtain

$$
\log \overline{\chi(\lambda)}=g M \operatorname{arcsinh}^{2} \sqrt{e^{i \lambda}-1}-\frac{M^{2}}{\beta}\left(3 \log \frac{\operatorname{arcsinh} \sqrt{e^{i \lambda}-1}}{\sqrt{e^{i \lambda}-1}}+\frac{1}{2} i \lambda\right)+O\left(M^{3}\right) .
$$

Note that for $M \ll g$ (short time limit), $\log \overline{\chi(\lambda)}$ reduces to $\overline{\log \chi(\lambda)}$. We expand $\log \overline{\chi(\lambda)}$ in terms of $\lambda$ to see features of $\overline{P(Q(t))}$ :

$$
\log \overline{\chi(\lambda)}=g M(i \lambda)+\left(\frac{g}{3} M+\frac{2}{15 \beta} M^{2}\right) \frac{(i \lambda)^{2}}{2 !}+\left(\frac{g}{15} M+\frac{2}{315 \beta} M^{2}+O\left(M^{3}\right)\right) \frac{(i \lambda)^{3}}{3 !}+\cdots .
$$

The first expansion coefficient shows that $\overline{\langle Q(t)\rangle}=Q_{0}=g M$, which is trivial. The second expansion coefficient, $\overline{\left\langle Q^{2}(t)\right\rangle}-\overline{\langle Q(t)\rangle}^{2}=\overline{\left\langle\left\langle Q^{2}(t)\right\rangle\right\rangle}+\operatorname{var}(\langle Q(t)\rangle)=Q_{0} / 3+(2 / 15 \beta) M^{2}$ indicates that the peak width of $\overline{P(Q(t))}$ has two contributions. The first contribution is related to the noise power, and the second one to the universal conductance fluctuations because $\operatorname{var}(\langle Q(t)\rangle)$ is proportional to the variance of the conductance. (The factor $2 / 15 \beta$ is precisely the variance of the dimensionless conductance.) Note that as $t \rightarrow \infty$, the second contribution becomes dominant over the first one. It can be shown that the $k$-th order expansion coefficient contains $k$ different contributions and at long time limit, the most 
dominant contribution, which is proportional to $M^{k}$, is related to the $k$-th cumulant of the conductance fluctuations. From this analysis we see that the behavior of $\overline{\chi(\lambda)}$ for large $t$ is governed by the conductance fluctuations instead of the current fluctuations.

As a short remark, we report that the continuum approximation calculation of $\log \overline{\chi(\lambda)}$, as suggested by Muttalib and Chen [15], produces the same result as Eq. (21) up to $M^{2}$ order.

In summary, we examine the counting statistics of charge to study the low temperature current fluctuations. By calculating the characteristic function of the probability distribution $P(Q(t))$, we find that $P(Q(t))$ has a Gaussian peak at $Q_{0}$ with $\langle\langle Q(t)\rangle\rangle=Q_{0} / 3$ and we also find that the tails of $P(Q(t))$ are different from the tails of Gaussian and classical Poisson distribution. By studying the variances of the cumulants, we establish that even though the peak location of $P(Q(t))$ varies from sample to sample due to universal conductance fluctuations, the peak shape of $P(Q(t))$ is universal in the metallic regime, and that the sample to sample variations show up only at the tails of $P(Q(t))$. 


\section{REFERENCES}

[1] J. B. Johnson, Phys. Rev. 29, 367 (1927) and H. Nyquist, Phys. Rev. 32, 110 (1928).

[2] R. Landauer, in Localization, Interaction and Transport Phenomena, eds. B. Kramer, G. Bergmann and Y. Bruynsraede (Springer, Heidelberg, 1985) Vol 61.

[3] G. B. Lesovik, Pis'ma Zh. Eksp. Teor. Fiz. 49, 513 (1989) [JETP Lett. 49, 592 (1989)].

[4] B. Yurke and G. P. Kochanski, Phys. Rev. B 41, 8184 (1990).

[5] M. Büttiker, Phys. Rev. Lett. 65, 2901 (1990).

[6] For a review see: A. D. Stone, P. A. Mello, K. A. Muttalib, and J.-L. Pichard, in Mesoscopic Phenomena in Solids, Edited by B. L. Al'tshuler, P. A. Lee, and R. A. Webb (North-Holland, Amsterdam, 1991).

[7] C. W. J. Beenakker and M. Büttiker, Phys. Rev. B 46, 1889 (1992).

[8] L. S. Levitov and G. B. Lesovik, JETP Lett. 58 (3), 230 (1993).

[9] P. A. Mello, P. Pereyra, and N. Kumar, Ann. Phys. 181, 290 (1988).

[10] The spin degeneracy is ignored. To include the degeneracy, $M$ has to be multiplied by 2. Also the positivity of $M$ is assumed. If $M$ is negative, Eq. (5) has to be complex conjugated with $M$ replaced by its absolute value.

[11] M. J. M. de Jong and C. W. J. Beenakker, Phy. Rev. B 46, 13400 (1992).

[12] C. W. J. Beenakker and B. Rejaei, Phys. Rev. Lett. 71, 3689 (1993).

[13] J. T. Chalker and A. M. S. Macêdo, Phys. Rev. Lett. 71, 3693 (1993).

[14] C. W. J. Beenakker, Phys. Rev. Lett. 70, 1155 (1993).

[15] K. A. Muttalib and Y. Chen, preprint (cond-mat/papers/9405068@babbage.sissa.it). 